\title{
Disparities in survival for elders with disabilities: implication for long-term care insurance Disparities in survival for elders with disabilities
}

Cynthia Chen ( $\square$ ephchc@nus.edu.sg)

National University of Singapore https://orcid.org/0000-0002-1947-8069

Jue Tao Lim

National University of Singapore

Ngee Choon Chia

National University of Singapore

Lijia Wang

National University of Singapore

Ming Zhe Chong

National University of Singapore

Angela Cheong

National University of Singapore

Ngan Phoon Fong

National University of Singapore

Boon Yeow Tan

St Luke's Hospital

\section{Edward Menon}

St Andrew's Community Hospital

Chye Hua Ee

Bright Vision Hospital

Kok Keng Lee

Bright Vision Hospital

Kin Ming Chan

Ang Mo Kio - Thye Hua Kwan Hospital

Stefan Ma

Ministry of Health

Kelvin Bryan Tan

Ministry of Health

Gerald CH Koh

National University of Singapore

Research article

Keywords: Disabilities; Elderly, Long-term care, Insurance, Singapore, Cox proportional-hazard regression, Survival, Policy 
Posted Date: August 28th, 2019

DOI: https://doi.org/10.21203/rs.2.10967/v1

License: (c) (i) This work is licensed under a Creative Commons Attribution 4.0 International License. Read Full License 


\section{Abstract}

Background Singapore is ageing rapidly and has one of the longest life expectancies in the World. Long-term care also represents one of the largest financial risks of the elderly. ElderShield, a long-term care insurance scheme provides payouts up to six years for elders with severe disability - having three or more ADL disabilities. The six years coverage might be insufficient due to increases in the duration of disability and longer life expectancy. Methods In this paper, we studied all elders admitted to community hospitals in Singapore from 1996 to 2005 , linked with national death records until December 2011. We modelled their survival risk after discharge from community hospitals using Cox proportional-hazards regression, taking into account ADL disability, health status and other socio-demographic covariates. Results Individuals with higher ADL disability counts have a higher hazard ratio (HR: 1.28,1.74,1.95 for mild, moderate and severe disability respectively compared to those with no disability), males had a higher hazard ratio compared to females (HR:1.40), older age (HR:1.87), being in a higher subsidy ward class (HR: 1.09 and 1.12 for moderate and high subsidy respectively compared to no/low subsidy) and had greater comorbidity burden (HR: 1.17, 1.14, 1.39 for mild, moderate and severe comorbidity burden respectively compared to those with no comorbidity burden). We also found that survival probabilities for individuals with two disabilities were not statistically different from those with 3+ ADL disabilities. In addition, more elders with severe disability were living longer than six years. Conclusions This has implications on long-term care policies in the context of ElderShield, as the disability insurance use severe ADL disabilities as the qualifying criteria for payout, capped at six years. While the CareShield Life Plan achieves more coverage than ElderShield, the payment into the plan also increases. Nevertheless, ensuring fiscal sustainability and affordable premium is challenging for any government, especially with Singapore's rapidly ageing demography. We conclude that the expansion of coverage from six years to life is timely as Singaporeans are living longer with ADL disability. These payouts will be helpful for the disabled elders and their family, protecting them against some financial risk.

\section{Introduction}

Long-term care (LTC) represents one of the largest financial risks of the elderly worldwide. A rapidly ageing demographic has led to a rise in long-term care expenditure, resource utilisation and the number of LTC workers in OECD countries from 2000 to 2017. For Japan, the number of LTC workers almost tripled from 727,339 to 2,028,341, from 2000 to 2017. In the United States, the number of LTC workers increased from 1,995,395 in 2005 to 2,791,842 in 2016. In the OECD, 30 countries with LTC represent 1.4\% of public spending on average in 2017. (Organisation for Economic Co-operation and Development, 2018) In Singapore, the number of intermediate and LTC facilities saw minor increases from 61 to 66 in 2006 to 2015 (GovTech, 2018) and might be insufficient to meet future needs.

Singapore is projected to take 27 years to transition from an ageing society in 1999 with only $7 \%$ seniors to a 'superaged society' with more than $20 \%$ seniors in 2026 , surpassing Japan, China, Germany and the United States, which took or will take 36, 32, 76 and 86 years to make the transition respectively. (East Asia Forum, 2015; Tan Teck Boon, 2015) While she has one of the lowest healthcare spending per capita among the developed countries and longest life expectancies in the world, (Bank, 2017) it is unclear if she can continue to keep cost low without compromising on health outcomes. 
Questions regarding health care financing, cost containment, successful ageing and resource utilisation have been an increasing concern. With a rapidly ageing population, the risk of functional disability in many elderly persons will increase, resulting in an increased demand for long-term care and higher medical spending. (Broe et al., 2002; Edelbrock et al., 2003; Wu et al., 2013) The economic burden of functional disability and long-term care has also significantly driven healthcare expenditure among the elderly. (Fried et al., 2001; James Lubitz, 2003; Picco et al., 2016; Spillman, 2004) In Taiwan, the government reported a 7\% increase in the prevalence of disabilities among older adults (from 29.6\% in 1997 to $36.6 \%$ in 2010). (Ministry of the Interior, 2010) In addition, hospitalisation rates for elders in Taiwan with functional disabilities were found to be much higher than the general population. (Lin et al., 2011) Japanese medical spending was at least three times higher for disabled individuals in performing selfcare, compared to their respective peers with no functional disabilities. (Tsuji et al., 1999)

Functional disabilities are expected to increase among the elderly population. (United Nations Department of Economic and Social Affairs, 2015) Furthermore, an increasingly ageing population makes this issue far more prominent and raises many questions regarding the sustainability of healthcare financing on both the societal and individual level. In Singapore, ElderShield is a long-term care insurance scheme that pays out (S\$400 per month) for a maximum period of six years for those with severe disability. This encompasses only basic financial protection for long-term care. Individuals may also purchase supplements for additional coverage on this scheme. From 2020, the plan will be replaced with CareShield Life, which removes the six years cap and will provide lifetime cash payouts for an individual with severe disability. In addition, the payout will be increased to between S\$600 and $\$ 1,200$.

The recent proliferation of long-term care, technological improvement and availability of assistive devices may have contributed to a large reversal in later life functional outcomes. (Manton et al., 2006) However, not all can afford technological improvements or invest in healthcare (Goldman and Lakdawalla, 2001) and this necessitates universal long-term care insurance schemes to hedge the public against later life functional disability. Hedging against potential financial risks in long-term care is important as long-term care represents a vast portion of old-age spending. Elders may be present-bias, thus not able to evaluate eventual frailty or disability, events that happen with low probability but high-cost (similar to the onset of chronic diseases). They may also avoid paying for long-term care insurance and rely on family members to provide support later in life or utilize other informal care services. (Norton, 2000) Literature also suggests that long-term care may be essential for individuals who are severely disabled, helping them improve their quality of life and mental health, and delay mortality through various integrated long-term care programmes (Kiely et al., 2000; Le Roux and Kemp, 2009; Schulz et al., 2004). However, those who are in poor health are more likely to be unable to afford health and long-term care to improve living in old age. (Deaton and Paxson, 2001; Kawachi et al., 1999; Kennedy et al., 1998) In addition, females tend to have a lower lifetime income compared to males, are also more likely to live longer and possess experience functional disability later in life. (Davidson et al., 2011) This begs the question of whether long-term care insurance achieves sufficient coverage among different groups within the Singapore healthcare system.

In this paper, a historical national population of individuals admitted to community hospitals was studied. We analyzed how time to mortality changes on discharge for individuals of different functional disability counts, and 
adjusted for economic, socio-demographic and health status. Higher hazard ratios were associated with individuals who are male, more functionally disabled, Malay, have a greater comorbidity burden and higher subsidy ward. We discuss our findings in the context of ElderShield and CareShield and the low long-term care coverage in Singapore among disabled elders.

\section{Methods}

\subsection{Study Population}

The study population $(n=12,804)$ was a historical national cohort taken from a database of all patients admitted to all community hospitals for rehabilitation in Singapore between 1996 and 2005. Community hospitals are rehabilitation hospitals that cater to patients who are fit for discharge from acute hospitals but require inpatient convalescent or subacute rehabilitative care before returning to a final domiciliary site. We excluded patients who died during their hospital stay in the community hospital $(n=24)$. Inclusion criteria for this study were first admission to community hospitals for inpatient rehabilitation from acute functional disability (e.g. stroke and hip fractures) and functional disability assessed at discharge.

The study was approved by the National University of Singapore Institutional Review Board (NUS-IRB) and ethics committees of Ang Mo Kio Thye Hua Kwan Hospital, Bright Vision Hospital, St Andrew's Community Hospital and St Luke's Hospital. Written informed consent of the patient was waived by approving NUS-IRB. The corresponding author and all research nurses have taken the oath of confidentiality under Singapore's Official Secrets Act and only the minimum number of research personnel had access to the de-identified dataset.

\subsection{Covariate}

Covariates were controlled to elicit the specific effects of time to mortality upon discharge including sociodemographic variables such as age, gender (female, male), ethnicity (Chinese, Malay, Indian, others), marital status (single, married, widowed, separated or divorced), the Charlson comorbidity index (CCMI) group (0, 1-3, 4-6 and 7+), subsidy ward class and length of hospital stay. Hospitalization subsidy level served as a proxy measure for socioeconomic status (SES). In Singapore, $81 \%$ of public hospitals' beds (B2 and C class) are highly subsidized. (Ministry of Health Singapore, 2004) B2+and B1 class lower subsidy and A class have no subsidy.

Comorbidity burden was measured using the CCMI. (Charlson et al., 1987) Comorbidity data was manually extracted by trained medical practitioners by reviewing the patients' medical problem list and medical records. The 
CCMI measures the severity of medical illnesses and comprises of 19 different categories. The overall CCMI score is a sum of weighted scores of diseases. A higher score reflects an individual's greater cumulative disease burden. We categorized index scores into four groups: no comorbidity (0), low comorbidity (1-3), moderate comorbidity (46) and high comorbidity (7-16). The Charlson comorbidity score also has been shown to have a high inter-rater reliability of a kappa score of 0.93. (Bernardini et al., 2004)

\subsection{Exposures of interest}

The number of functional disabilities was measured according to activities of daily living (ADL) measurements at discharge. These include an individual's ability to conduct washing, feeding, toileting, mobility, transferring and dressing. As individuals only qualify for ElderShield with three or more functional disabilities, we classified individuals with the number of disabilities to be $0,1,2$ and $3+$. We defined those with one disability as having mild disability, two disabilities as moderate disability, and having three or more disabilities as severe disability. For bivariable analyses, Chi-square tests were performed for categorical variables, Kruskal-Wallis tests for length of stay and ANOVA test was performed for age across disability groups.

\subsection{Outcome of interest}

Survival time from discharge was computed by taking the difference of date of discharge with the time of death from the Ministry of Health death registry. All study subjects were censored at 31 December 2011 . Survival status was cross-referenced with the Singapore's National Death Registry. As ElderShield subsidy cutoffs at 6 years, we also recategorized survival time to 6 years or more and less than 6 years among those who died.

\subsection{Multivariable Analysis}

Cox proportional-hazards regressions were run with severity of ADL disability, gender, age, race, marital status, subsidy ward class and comorbidity burden added to our specification sequentially. This was done to identify predictors of mortality. Schoenfeld residuals were used to test the proportional hazards (PH) assumption after model fitting. Stratifying factors such as primary diagnosis at admission, community hospitals and year of admission were included in the Cox model. All analyses were performed using STATA version 13 (StataCorp LP, USA) with the significance level set at 0.05 . Statistical significance for all analysis was set at $5 \%$ level. 


\section{Results}

Bivariable analysis was conducted on severity of functional disability and was found to be statistically significant by length of stay, ethnicity, gender, comorbidity burden, subsidy ward class and marital status. The sample length of inpatient stay was 32 days. Patients with mild disability had higher inpatient length of stay of 34 days. In addition, patients who were more likely to have severe functional disability were males $(24.4 \% \mathrm{vs} .22 .7 \%$, $p=0.036)$, Malays compared to the Chinese ( $31.5 \%$ vs. $22.7 \%$, p<0.001), had greater comorbidity burden ( $6.9 \%$ vs. $3.7 \%$ for severe vs no comorbidity burden, $\mathrm{p}<0.001)$, were admitted to lower subsidy ward $(21.2-32.2 \%$ vs. $18.2 \%$ in Class $A-B 2+$ vs Class $B 2, p<0.001)$, married compared to the singles $(25.4 \%$ vs. $13.3 \%, p<0.001)$. There was no significant difference in the mean age $(p=0.216)$ across disabled groups. These results stratified by gender was reported in Appendix Table 1a and 1b.

Table 1: Characteristics of patients by severity of functional disability 


\begin{tabular}{|c|c|c|c|c|c|c|}
\hline & Total & No $(0)$ & Mild (1) & $\begin{array}{l}\text { Moderate } \\
\text { (2) }\end{array}$ & Severe (3+) & P-value \\
\hline & $(n=12804)$ & $(n=7512)$ & $(n=1532)$ & $(n=763)$ & $(n=2997)$ & \\
\hline Age (Years), Mean (SE) & $73.0(11.5)$ & $72.3(11.6)$ & 73.5(11.6) & $74.9(11.0)$ & $74.5(11.4)$ & $0.216^{\dagger}$ \\
\hline $\begin{array}{l}\text { Length of Stay, Median } \\
\text { (IQR) }\end{array}$ & $32(20-47)$ & $31(21-47)$ & $34(23-49)$ & $31(18-46)$ & $31(17-49)$ & $<0.001^{\ddagger}$ \\
\hline \multicolumn{7}{|l|}{ Ethnicity, n (\%) } \\
\hline Chinese & $\begin{array}{l}11293 \\
(88.1 \%)\end{array}$ & $\begin{array}{l}6738 \\
(59.7 \%)\end{array}$ & $\begin{array}{l}1335 \\
(11.8 \%)\end{array}$ & 653 (5.8\%) & $\begin{array}{l}2567 \\
(22.7 \%)\end{array}$ & $<0.001^{\S}$ \\
\hline Malay & 848 (6.6\%) & 395 (46.6\%) & $\begin{array}{l}115 \\
(13.6 \%)\end{array}$ & 71 (13.6\%) & 267 (31.5\%) & \\
\hline Indian & $518(4.1 \%)$ & $289(55.7 \%)$ & $66(12.7 \%)$ & $32(6.2 \%)$ & $132(25.4 \%)$ & \\
\hline Others & $145(1.1 \%)$ & 90 (62.5\%) & $16(12.0 \%)$ & $7(6.0 \%)$ & $31(23.4 \%)$ & \\
\hline \multicolumn{7}{|l|}{ Gender, n (\%) } \\
\hline Male & $\begin{array}{l}5341 \\
(41.7 \%)\end{array}$ & $3076(57.6 \%)$ & $624(11.7 \%)$ & $338(6.3 \%)$ & $1303(24.4 \%)$ & $0.036^{\S}$ \\
\hline Female & $\begin{array}{l}7463 \\
(58.3 \%)\end{array}$ & $4436(59.4 \%)$ & $908(12.2 \%)$ & $424(5.7 \%)$ & $1694(22.7 \%)$ & \\
\hline \multicolumn{7}{|l|}{$\begin{array}{l}\text { Comorbidity burden, } \mathrm{n} \\
(\%)\end{array}$} \\
\hline No $(0)$ & $2377(18.6 \%)$ & $1745(73.4 \%)$ & 263(11.1\%) & $87(3.7 \%)$ & $282(11.9 \%)$ & $<0.001^{\S}$ \\
\hline Mild (1 to 3 ) & $5878(45.9 \%)$ & $3463(58.9 \%)$ & $717(12.2 \%)$ & $374(6.4 \%)$ & $1324(22.5 \%)$ & \\
\hline Moderate (4 to 6) & $4012(31.3 \%)$ & $2035(50.7 \%)$ & 472(11.8\%) & $265(6.6 \%)$ & $1240(30.9 \%)$ & \\
\hline Severe $(7+)$ & $537(4.19 \%)$ & $269(50.1 \%)$ & $80(14.9 \%)$ & $37(6.9 \%)$ & $151(28.1 \%)$ & \\
\hline
\end{tabular}

\section{Subsidy ward class, $\mathbf{n}$}

(\%)

\begin{tabular}{lllllll}
$\begin{array}{l}\text { No/low (A - B2+ } \\
\text { class) }\end{array}$ & $1207(9.4 \%)$ & $716(59.3 \%)$ & $161(13.3 \%)$ & $74(6.1 \%)$ & $256(21.2 \%)$ & $<0.001 \S$ \\
\hline Moderate (B2 class) & $4478(35.0 \%)$ & $2318(51.8 \%)$ & $455(10.2 \%)$ & $259(5.8 \%)$ & $1446(32.3 \%)$ & \\
\hline High (C class) & $7119(55.6 \%)$ & $4478(62.9 \%)$ & $916(12.9 \%)$ & $430(6.0 \%)$ & $1295(18.2 \%)$
\end{tabular}

\section{Marital Status, n (\%)}

\begin{tabular}{lllllll} 
Single & $1099(8.6 \%)$ & $798(72.6 \%)$ & $109(9.9 \%)$ & $46(4.2 \%)$ & $146(13.3 \%)$ & $<0.001 \S$ \\
\hline Married & $5483(42.8 \%)$ & $3086(56.3 \%)$ & $649(11.8 \%)$ & $358(6.5 \%)$ & $1390(25.4 \%)$ \\
\hline Widowed & $5853(45.7 \%)$ & $3373(57.6 \%)$ & $736(12.6 \%)$ & $344(5.9 \%)$ & $1400(23.9 \%)$ \\
\hline Separated/Divorced & $369(2.9 \%)$ & $255(69.1 \%)$ & $38(10.3 \%)$ & $15(4.1 \%)$ & $61(16.5 \%)$
\end{tabular}


${ }^{\dagger}$ ANOVA test; ${ }^{\ddagger}$ Kruskal-Wallis; $§$ Chi-square test

We found statistically significant difference among various covariates and mortality (Table 2). Mortality risk was significantly associated with older age, longer length of hospital stay, worse functional disability, ethnicity, gender, CCMI score and higher subsidy ward class. Those with lower mortality risk were females compared to males (64.9\% vs. $69.7 \%$, $p<0.001)$, had shorter length Chinese compared to Malay (66.9\% vs. $69.1 \%, p=0.009)$, had lower comorbidity burden ( $53.4 \%$ vs. $83.1 \%$ in 0 vs. $7+$ CCMI score, $p<0.001$ ), had no functional disability compared to those with severe disability ( $58.7 \%$ vs. $82.5 \%, p<0.001)$. Old age was strongly associated with mortality $(p<0.001)$. We found no significant difference in marital status across groups $(p=0.388)$. 
Table 2: Characteristics of patients by time to mortality

Variables

Mortality

\begin{tabular}{|c|c|c|c|c|c|c|}
\hline & Total & $\begin{array}{l}\text { Died at time } \\
\text { of censoring }\end{array}$ & $\begin{array}{l}\text { Died, } \geq 6 \\
\text { years after } \\
\text { admission }\end{array}$ & $\begin{array}{l}\text { Died, < } 6 \\
\text { years after } \\
\text { admission }\end{array}$ & $\begin{array}{l}\text { Alive with } \\
\geq 6 \text { years } \\
\text { after } \\
\text { admission }\end{array}$ & P-value \\
\hline & $(n=12804)$ & $(n=8565)$ & $(n=2034)$ & $(n=6531)$ & $(n=4239)$ & \\
\hline Age, mean (SE) & 73.0(11.5) & 75.6 (10.3) & $73.6(10.1)$ & $76.3(10.2)$ & $67.7(12.2)$ & $<0.001^{\dagger}$ \\
\hline $\begin{array}{l}\text { Length of Stay, } \\
\text { median (IQR) }\end{array}$ & $32(20-47)$ & $32(20-48)$ & $34(21-51)$ & $31(19-47)$ & $31(20-46)$ & $<0.001^{\ddagger}$ \\
\hline \multicolumn{7}{|l|}{$\begin{array}{l}\text { Severity of } \\
\text { disability, } n(\%)\end{array}$} \\
\hline $\begin{array}{l}\text { No (0 ADL } \\
\text { disability) }\end{array}$ & $\begin{array}{l}7512 \\
(58.7 \%)\end{array}$ & $4406(58.7 \%)$ & 1195(15.9\%) & $3211(42.7 \%)$ & $3106(41.3 \%)$ & $<0.001^{\S}$ \\
\hline $\begin{array}{l}\text { Mild (1 ADL } \\
\text { disability) }\end{array}$ & $\begin{array}{l}1532 \\
(11.9 \%)\end{array}$ & 1077(70.3\%) & $256(16.7 \%)$ & $821(53.6 \%)$ & $455(29.7 \%)$ & \\
\hline $\begin{array}{l}\text { Moderate (2 ADL } \\
\text { disabilities) }\end{array}$ & $763(6 \%)$ & $608(79.7 \%)$ & $106(13.9 \%)$ & $502(65.8 \%)$ & $155(20.3 \%)$ & \\
\hline $\begin{array}{l}\text { Severe (3+ ADL } \\
\text { disabilities) }\end{array}$ & $2997(23.4)$ & $2474(82.5 \%)$ & $477(15.9 \%)$ & 1997(66.6\%) & $523(17.5 \%)$ & \\
\hline \multicolumn{7}{|l|}{ Ethnicity, n(\%) } \\
\hline Chinese & $\begin{array}{l}11293 \\
(88.1 \%)\end{array}$ & 7555(66.9\%) & 1833(16.2\%) & $5722(50.7 \%)$ & 3738(33.1\%) & $0.009 \S$ \\
\hline Malay & 848 (6.6\%) & $586(69.1 \%)$ & 108(12.7\%) & $478(56.4 \%)$ & $262(30.9 \%)$ & \\
\hline Indian & $\begin{array}{l}518 \\
(4.046 \%)\end{array}$ & $332(64.1 \%)$ & $70(13.5 \%)$ & $262(50.6 \%)$ & 186(35.9\%) & \\
\hline Others & 145 (1.07\%) & $92(63.4 \%)$ & $23(15.9 \%)$ & $69(47.6 \%)$ & $53(36.6 \%)$ & \\
\hline \multicolumn{7}{|l|}{ Gender, n(\%) } \\
\hline Male & $\begin{array}{l}5341 \\
(41.7 \%)\end{array}$ & $3721(69.7 \%)$ & $726(13.6 \%)$ & 2995(56.1\%) & $1620(30.3 \%)$ & $<0.001^{\S}$ \\
\hline Female & $\begin{array}{l}7463 \\
(58.3 \%)\end{array}$ & $4844(64.9 \%)$ & $1308(17.5 \%)$ & $3536(47.4 \%)$ & $2619(35.1 \%)$ & \\
\hline
\end{tabular}

\section{Comorbidity}

Burden, $n(\%)$

\begin{tabular}{lllllll} 
No (0) & $2377(18.6 \%)$ & $1270(53.4 \%)$ & $354(14.9 \%)$ & $916(38.5 \%)$ & $1107(46.6 \%)$ & $<0.001 \S$ \\
\hline Mild (1 to 3) & $5878(45.9 \%)$ & $3886(66.1 \%)$ & $972(16.5 \%)$ & $2914(49.6 \%)$ & $1992(33.9 \%)$ \\
\hline Moderate (4 to 6) & $4012(31.3 \%)$ & $2963(73.9 \%)$ & $662(16.5 \%)$ & $2301(57.4 \%)$ & $1049(26.1 \%)$ \\
\hline Severe (7+) & $537(4.2 \%)$ & $446(83.1 \%)$ & $46(8.6 \%)$ & $400(74.5 \%)$ & $91(16.9 \%)$
\end{tabular}

Subsidy Ward 


\begin{tabular}{|c|c|c|c|c|c|c|}
\hline \multicolumn{7}{|l|}{ Class, n (\%) } \\
\hline $\begin{array}{l}\text { No/low (Class :A - } \\
\mathrm{B} 2+\text { ) }\end{array}$ & $1207(9.4 \%)$ & $783(64.9 \%)$ & $245(20.3 \%)$ & $538(44.6 \%)$ & 424(35.1\%) & $<0.001^{\S}$ \\
\hline $\begin{array}{l}\text { Moderate (Class: } \\
\text { B2) }\end{array}$ & $4478(35.0 \%)$ & $3117(69.6 \%)$ & $876(19.6 \%)$ & $2241(50.0 \%)$ & $1361(30.4 \%)$ & \\
\hline High (Class: C) & $7119(55.6 \%)$ & $4665(65.5 \%)$ & $913(12.8 \%)$ & $3752(52.7 \%)$ & $2454(34.5 \%)$ & \\
\hline \multicolumn{7}{|l|}{ Marital Status, n(\%) } \\
\hline Single & 1099(8.6\%) & $581(52.9 \%)$ & $154(14.0 \%)$ & $427(38.9 \%)$ & $518(47.1 \%)$ & $0.388^{\S}$ \\
\hline Married & $5483(42.8 \%)$ & $3544(64.6 \%)$ & $823(15.0 \%)$ & $2721(49.6 \%)$ & 1939(35.4\%) & \\
\hline Widowed & $5853(45.7 \%)$ & $4238(72.4 \%)$ & $1010(17.3 \%)$ & $3228(55.2 \%)$ & $1615(27.6 \%)$ & \\
\hline Separated/Divorced & $369(2.9 \%)$ & $202(54.7 \%)$ & $47(12.7 \%)$ & $155(42.0 \%)$ & $167(45.3 \%)$ & \\
\hline
\end{tabular}

Adjusting for all other confounders, individuals with higher ADL disability counts have a higher hazard ratio (HR:

$1.28,1.74,1.95$ for mild, moderate and severe disability respectively compared to those with no disability), males had a higher hazard ratio compared to females (HR:1.40), older age (HR:1.87), Malay had higher hazard ratio compared to Chinese (HR:2.89), being in a higher subsidy ward class (HR: 1.09 and 1.12 for moderate and high subsidy respectively compared to no/low subsidy) and had greater comorbidity burden (HR: 1.17, 1.14, 1.39 for mild, moderate and severe comorbidity burden respectively compared to those with no comorbidity burden).

Table 3. Cox proportional-hazards regression of survival time 
Outcome: Survival Time

\begin{tabular}{|c|c|c|c|}
\hline \multirow[t]{2}{*}{ Variables } & \multicolumn{3}{|l|}{ Hazard Ratio` } \\
\hline & Model (1) & Model (2) & Model (3) \\
\hline \multicolumn{4}{|l|}{ Severity of disability } \\
\hline No disability & 1.00 & 1.00 & 1.00 \\
\hline Mild (1 ADL disability) & $1.38(1.29,1.48)^{\star \star \star}$ & $1.33(1.24,1.42)^{\star \star \star}$ & $1.28(1.19,1.37)^{\star}$ \\
\hline Moderate (2 ADL disabilities) & 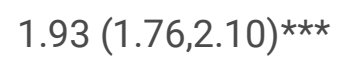 & 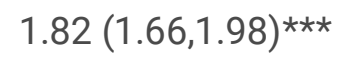 & 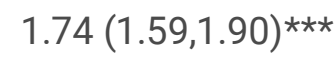 \\
\hline Severe (3+ ADL disabilities) & 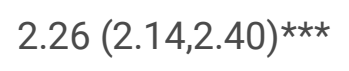 & $2.06(1.94,2.18)^{\star \star \star}$ & $1.95(1.84,2.07)^{\star \star \star}$ \\
\hline \multicolumn{4}{|l|}{ Gender } \\
\hline Female & & 1.00 & 1.00 \\
\hline Male & & 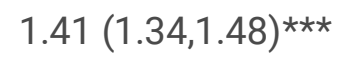 & 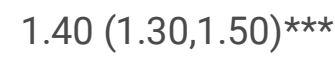 \\
\hline Age & & 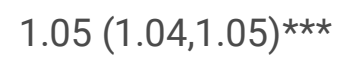 & $1.87(1.73,2.03)^{\star \star \star}$ \\
\hline \multicolumn{4}{|l|}{ Race } \\
\hline Chinese & & 1.00 & 1.00 \\
\hline Malay & & $1.24(1.14,1.36)^{\star \star \star}$ & $2.89(2.56,3.26)^{\star \star \star}$ \\
\hline Indian & & $1.07(0.96,1.20)$ & $1.39(1.32,1.47)$ \\
\hline Others & & $0.93(0.75,1.15)$ & $1.05(1.04,1.05)$ \\
\hline \multicolumn{4}{|l|}{ Marital Status } \\
\hline Single & & 1.00 & 1.00 \\
\hline Married & & 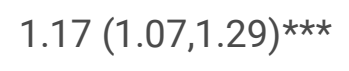 & $1.22(1.11,1.33)^{\star}$ \\
\hline Widowed & & $1.20(1.09,1.32)^{\star \star \star}$ & $1.03(0.92,1.15)^{\star \star}$ \\
\hline Separated/Divorced & & $1.22(1.03,1.44)^{\star}$ & $0.93(0.75,1.15)^{\star}$ \\
\hline \multicolumn{4}{|l|}{ Subsidy Ward Class, n (\%) } \\
\hline No/low (Class: A - B2+) & & 1.00 & 1.00 \\
\hline Moderate (Class: B2) & & $1.11(1.02,1.21)^{\star \star}$ & $1.09(0.99,1.20) *$ \\
\hline High (Class: C) & & $1.36(1.25,1.48)^{\star \star \star}$ & $1.12(1.02,1.24)^{\star \star}$ \\
\hline \multicolumn{4}{|l|}{ Comorbidity Burden } \\
\hline No $(0)$ & & & 1.00 \\
\hline Mild (1 to 3 ) & & & 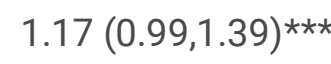 \\
\hline Moderate (4 to 6) & & & $1.14(1.05,1.24)^{\star \star \star}$ \\
\hline Severe $(7+)$ & & & $1.39(1.28,1.52)^{\star \star \star}$ \\
\hline
\end{tabular}


* $0.05<$ P-value $\leq 0.1 ; * * 0.001<$ P-value $<0.05 ; * \star *$ P-value $<0.001$

' Hazard ratios were reported using Cox proportional-hazard model. Models were stratified based on primary diagnosis at admission, community hospitals and year of admission. Multivariate models are adjusted for confounders such as gender, ethnicity, marital status, subsidy ward class and the Charlson Comorbidity index.

Cumulative hazards plots by classifying severity of ADL disability showed that gender-specific lines did not cross. Females had greater survival than males across all disability groups, indicating that they were more likely to live longer given the same level of ADL disability (Figure 1). In addition, there was no difference in survival probabilities for individuals with moderate disability (two disabilities) and severe disability (three or more ADL disabilities) within genders. These survival probabilities were adjusted for age, race, comorbidity burden, subsidy ward class, marital status and stratified by primary diagnosis, community hospital and year of admission.

Figure 1: Survival plots for gender by ADL disability ${ }^{\dagger}$

Figure 1 illustrates survival probabilities for males and females across time, according to number of functional disabilities $^{\dagger}$ using Cox proportional-hazards model, adjusted for age, race, comorbidity burden, subsidy ward class, marital status, primary diagnosis, community hospital and year of admission.

${ }^{\dagger}$ Female (red color), Male (blue color).

Severity of disability: No disability= 0 ADL disability (dotted line), Mild= 1 ADL Disability (dash line), $\underline{\text { Moderate }=2}$ ADL Disabilities (long-dash line), Severe= 3 or more ADL disabilities (solid line)

\section{Discussion}

Our results indicated that individuals with ADL disability have a higher hazards ratio compared to individuals with no ADL disability. Furthermore, we identified other covariates which may lead to quicker mortality onset. These include being in a minority race (Malay), being male and admitted to a higher subsidy ward class. This confirms previous studies on ADL disability, ethnicity, gender and socioeconomic status as drivers of mortality onset. (Clarke et al., 2008; Kennedy et al., 1998; Millan-Calenti et al., 2010; Serrano-Alarcón and Perelman, 2017) However, we found that of survival after discharge for elders with moderate disability to be comparable to elders with severe disability after discharge. This has implications on long-term care policies in the context of ElderShield and CareShield Life, as both disability insurance schemes use severe ADL disabilities as the qualifying criteria for 
payout. There might be sufficient room to expand coverage to individuals with two or more ADL disabilities with the existing long-term care insurance schemes in Singapore without huge premiums loading for contributors. While the CareShield Life Plan achieves more coverage than ElderShield, the payment into the plan also increases.

Nevertheless, ensuring fiscal sustainability and affordable premium is challenging for any government, especially with Singapore's rapidly ageing demography. Our results also indicate that females tend to survive longer than males controlling for ADL disability status. This is consistent with existing literature reporting that disabled females require more physical assistance as they live longer compared to disabled males. (Dubuc et al., 2011) Furthermore, females tend to have lower labor force participation as well as lower pay in their time within the labor force. (Bank, 2017) However, both males and females receive the same payouts from the long-term care insurance schemes in Singapore. The issue is exacerbated with limited time payouts for ElderShield, which only covers disabled individuals for six years. This might not be socially optimal as they receive less income over time and require more assistance for long-term care in an equivalent portion of time compared to males. Disparities may even persist within preventive care for females and socioeconomically disadvantaged individuals (Katz and Hofer, 1994; Vaidya et al., 2012). Targeted policy formulation should factor gender disparities in comorbidities, functional disability and financial inputs into long-term care insurance. This will facilitate maintenance of functional and health status, as well as productive participation within society. (Stowe and Cooney, 2014) Access to healthcare services and preventive care are the first steps to retaining function and health as this makes a positive difference in healthy longevity and disability-free lifespan among elders. (Lin et al., 2004) Integrated long-term care as a secondary measure allows elders to retain independence, reduce hospitalization utilization and may even reverse functional disability among elders. (Challis et al., 1991; Eng et al., 1997) However, our results indicate that although Singapore's long-term care insurance requires equivalent input and provide equivalent assistance for both genders and among people with three or more disabilities, the marginal effort required for different groups may be different and assistance given for groups may vary across time.

\section{Limitations}

Usage of retrospective data and data incompleteness may have led to data entry biases in the dataset. Furthermore, the study was limited to investigating variables that the database included and did not consider other factors may also affect time to mortality (e.g. cognition, mental health, quality of life and healthcare decision making). Using subsidy ward class as a socioeconomic indicator may not be an exact proxy, but at most introduces random error to our model specification. As ADL disability was measured at the time of discharge, we were unable to update disability status. However, an argument against the insufficiency of coverage among individuals still holds as elders staying in the community tend to live longer compared to those who had been in an institutionalized setting. Our study underestimates the burden of disability as elders in nursing home were not routinely captured. Finally, the study was completed in an Asian population within a developed economy and an advanced healthcare system. Generalizing these findings to Western populations should be done with care.

\section{Conclusion}

To the best of the author's knowledge, this is the first study in South-East Asia which addresses ADL disability by counts and time to mortality after discharge for elders. In this paper, we put our results in the context of long-term care insurance within Singapore, one of the fastest ageing countries in the World. We conclude that the expansion of coverage from six years to life is timely as Singaporeans are living longer with ADL disability. These payouts will be helpful for the disabled elders and their family, protecting them against some financial risk.

Page $14 / 18$ 


\section{Disclaimer}

There are no disclaimers to report. The views and opinions expressed in this article are those of the authors and do not necessarily reflect those of funders or institutions of belonging.

\section{Declarations}

\section{Ethics approval and consent to participate}

The study was approved by the National University of Singapore Institutional Review Board (NUS-IRB) and ethics committees of Ang Mo Kio Thye Hua Kwan Hospital, Bright Vision Hospital, St Andrew's Community Hospital and St Luke's Hospital. Written informed consent of the patient was waived by approving NUS-IRB. The corresponding author and all research nurses have taken the oath of confidentiality under Singapore's Official Secrets Act and only the minimum number of research personnel had access to the de-identified dataset.

\section{Consent for publication}

Not Applicable

\section{Availability of data and material}

The datasets generated and/or analysed during the current study are not publicly available due the risk of violating patient privacy and trends in community level hospitals but are available from the corresponding author on reasonable request.

\section{Competing interests}

The author(s) declare(s) that they have no competing interests

\section{Funding}

This research is supported by the Singapore Ministry of Health's National Medical Research Council (HSRG0077/2017) and the Centre Grant Programme - Singapore Population Health Improvement Centre (NMRC/CG/C026/2017_NUHS). The funding sources had no role in the design and conduct of the study; collection, management, analysis, or interpretation of the data; preparation, review, or approval of the manuscript; and decision to submit the manuscript for publication.

\section{Authors' contributions}

Contributions to the conception: CC, CNC, GK. Designed the experiments: CC GK. Acquired and provided the data: AC NPF BYT EM CHE KKL KMC KBT GK. Statistical analysis: CC JTL. Interpretation of the results: CC JTL CNC WL MZC GK. Drafted the paper: CC JTL. All authors revised the article critically and gave final approval for publication. 


\section{Acknowledgements}

Not Applicable

\section{References}

Bank TW. 2017. Open Data. World Bank. 2017. Bernardini J, Callen S, Fried L, Piraino B. 2004. Inter-rater reliability and annual rescoring of the Charlson comorbidity index. Advances in peritoneal dialysis. Conference on Peritoneal Dialysis, vol. 20. 2004. Broe G, Grayson D, Waite L, Creasey H, Edelbrock D, Bennett H, Brooks W. Determinants of service use among the elderly: the Sydney older persons study. Australasian Journal on Ageing 2002;21; 61-66. Challis D, Darton R, Johnson L, Stone M, Traske K. An evaluation of an alternative to long-stay hospital care for frail elderly patients: II. Costs and effectiveness. Age and Ageing 1991;20; 245-254. Charlson ME, Pompei P, Ales KL, MacKenzie CR. A new method of classifying prognostic comorbidity in longitudinal studies: development and validation. Journal of chronic diseases 1987;40; 373-383. Clarke P, O'malley PM, Johnston LD, Schulenberg JE. Social disparities in BMI trajectories across adulthood by gender, race/ethnicity and lifetime socio-economic position: 1986-2004. International Journal of Epidemiology 2008;38; 499-509. Davidson PM, DiGiacomo M, McGrath SJ. The feminization of aging: how will this impact on health outcomes and services? Health Care for Women International 2011;32; 1031-1045. Deaton AS, Paxson C. 2001. Mortality, education, income, and inequality among American cohorts. Themes in the Economics of Aging. University of Chicago Press; 2001. Dubuc N, Dubois M-F, Raîche M, Hébert R. Meeting the home-care needs of disabled older persons living in the community: does integrated services delivery make a difference? BMC Geriatrics 2011;11; 67. East Asia Forum. 2015. Can a Rapidly Aging Singapore Stave Off Economic Disaster? vol. 2018. 2015. Edelbrock D, Waite LM, Broe GA, Grayson DA, Creasey $\mathrm{H}$. The relation between unpaid support and the use of formal health services: the Sydney Older Persons Study. Australasian Journal on Ageing 2003;22; 2-8. Eng C, Pedulla J, Eleazer GP, McCann R, Fox N. Program of Allinclusive Care for the Elderly (PACE): an innovative model of integrated geriatric care and financing. Journal of the American Geriatrics Society 1997;45; 223-232. Fried TR, Bradley EH, Williams CS, Tinetti ME. Functional disability and health care expenditures for older persons. Archives of Internal Medicine 2001;161; 2602-2607. Goldman D, Lakdawalla D. 2001. Understanding health disparities across education groups. Working Paper No. 8328. National Bureau of Economic Research; 2001. GovTech. 2018. Number of Intermediate and Long Term Care Facilities. vol. 2018. 2018. James Lubitz LC, Ellen Kramarow, and Harold Lentzner. Health, Life Expectancy, and Health Care Spending among the Elderly. New England Journal of Medicine 2003. Katz SJ, Hofer TP. Socioeconomic disparities in preventive care persist despite universal coverage: breast and cervical cancer screening in Ontario and the United States. Jama 1994;272; 530-534. Kawachi I, Kennedy BP, Wilkinson RG. Income Inequality and Healths. New Press New York; 1999. Kennedy BP, Kawachi I, Glass R, Prothrow-Stith D. Income distribution, socioeconomic status, and self rated health in the United States: multilevel analysis. Bmj 1998;317; 917-921. Kiely DK, Simon SE, Jones RN, Morris JN. The protective effect of social engagement on mortality in long-term care. Journal of the American Geriatrics Society 2000;48; 1367-1372. Le Roux MC, Kemp R. Effect of a companion dog on depression and anxiety levels of elderly residents in a long-term care facility. Psychogeriatrics 2009;9; 23-26. Lin EH, Katon W, Von Korff M, Rutter C, Simon GE, Oliver M, Ciechanowski P, Ludman EJ, Bush T, Young B. Relationship of depression and diabetes self-care, medication adherence, and preventive care. Diabetes care 2004;27; 2154-2160. Lin L-P, Lee J-T, Lin F-G, Lin P-Y, Tang C-C, Chu CM, Wu C-L, Lin J-D. Disability and hospital care expenses among national health insurance beneficiaries: Analyses of population-based data in Taiwan. Research in Developmental Disabilities 2011;32; 15891595. Manton KG, Gu X, Lamb VL. Change in chronic disability from 1982 to 2004/2005 as measured by long-term changes in function and health in the US elderly population. Proceedings of the National Academy of Sciences

Page $16 / 18$ 
2006;103; 18374-18379. Millan-Calenti JC, Tubío J, Pita-Fernández S, González-Abraldes I, Lorenzo T, FernandezArruty T, Maseda A. Prevalence of functional disability in activities of daily living (ADL), instrumental activities of daily living (IADL) and associated factors, as predictors of morbidity and mortality. Archives of Gerontology and Geriatrics 2010;50; 306-310. Ministry of Health Singapore M. Healthcare Services for the Elderly. An information booklet for healthcare professionals. 2004. Ministry of the Interior M. Statistical Yearbook of the Interior, 2010, 0418. The disabled population by age and grade. Republic of China: Department of statistics \{MOI, 2010 \#44\}. 2010. Norton EC. Long-term care. Handbook of Health Economics 2000;1; 955-994. Organisation for Economic Cooperation and Development O. OECD.Stat. 2018. Picco L, Achilla E, Abdin E, Chong SA, Vaingankar JA, McCrone P, Chua HC, Heng D, Magadi H, Ng LL. Economic burden of multimorbidity among older adults: impact on healthcare and societal costs. BMC Health Services Research 2016;16; 173. Schulz R, Belle SH, Czaja SJ, McGinnis KA, Stevens A, Zhang S. Long-term care placement of dementia patients and caregiver health and well-being. Jama 2004;292; 961-967. Serrano-Alarcón M, Perelman J. Ageing under unequal circumstances: a cross-sectional analysis of the gender and socioeconomic patterning of functional limitations among the Southern European elderly. International Journal for Equity in Health 2017;16; 175. Spillman BC. Changes in elderly disability rates and the implications for health care utilization and cost. The Milbank Quarterly 2004;82; 157-194. Stowe JD, Cooney TM. Examining Rowe and Kahn's concept of successful aging: Importance of taking a life course perspective. The Gerontologist 2014;55; 43-50. Tan Teck Boon. 2015. A super-aged Singapore: Policy implications for a Smart Nation, vol. 2018. Todayonline; 2015. Tsuji I, Kuwahara A, Nishino Y, Ohkubo T, Sasaki A, Hisamichi S. Medical cost for disability: a longitudinal observation of national health insurance beneficiaries in Japan. Journal of the American Geriatrics Society 1999;47; 470-476. United Nations Department of Economic and Social Affairs U. 2015. Global Status Report on Disability and Development. 2015. Vaidya V, Partha G, Karmakar M. Gender differences in utilization of preventive care services in the United States. Journal of Women's Health 2012;21; 140-145. Wu C-Y, Hu H-Y, Li C-P, Fang Y-T, Huang N, Chou Y-J. The association between functional disability and acute care utilization among the elderly in Taiwan. Archives of Gerontology and Geriatrics 2013;57; 177-183.

\section{Figures}




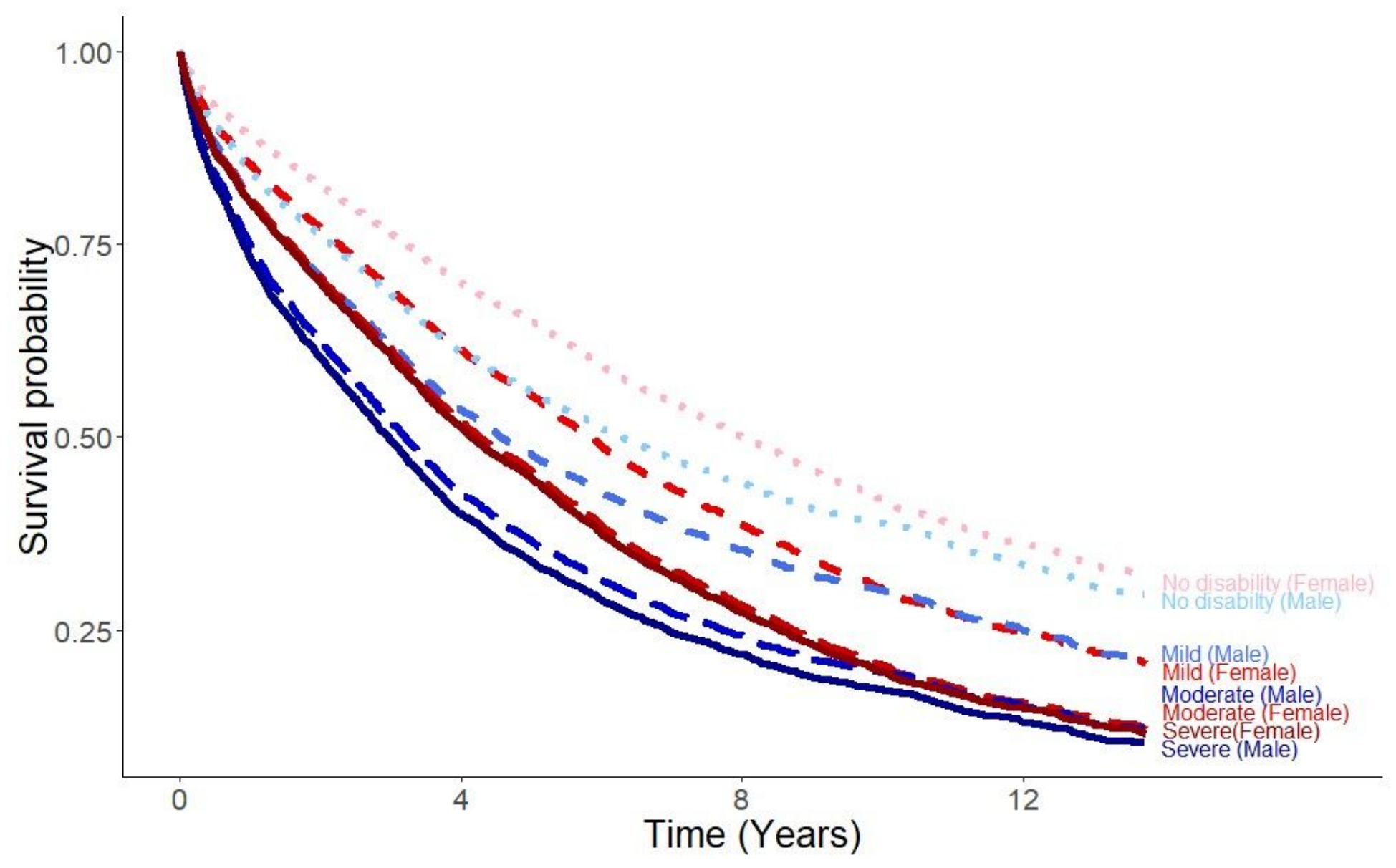

\section{Figure 1}

illustrates survival probabilities for males and females across time, according to number of functional disabilitiest using Cox proportional-hazards model, adjusted for age, race, comorbidity burden, subsidy ward class, marital status, primary diagnosis, community hospital and year of admission. † Female (red color), Male (blue color). Severity of disability: No disability= 0 ADL disability (dotted line), Mild= 1 ADL Disability (dash line), Moderate= 2 ADL Disabilities (long-dash line), Severe $=3$ or more ADL disabilities (solid line)

\section{Supplementary Files}

This is a list of supplementary files associated with this preprint. Click to download.

- supplement1.docx 